\title{
Hemangioma cavernoso como causa de hemorragia digestiva oculta manifiesta: reporte de caso
}

\section{A Case Report of Cavernous Hemangioma as a Cause of Occult Gastrointestinal Bleeding}

Diego Mauricio Gómez R., Juan Ramón Abello R., ${ }^{1}$ Alfredo Cienfuegos R., ${ }^{1}$ Gerardo Andrés Guzmán, ${ }^{1}$ Juliana Suárez C., ${ }^{1}$

Fredy Alexander León, ${ }^{1}$ William Salazar A., ${ }^{1}$ Ramiro Pinedo, ${ }^{2}$ Adrián Tabares, ${ }^{3}$ María Juliana Varela M. ${ }^{4}$

Especialista en gastroenterología y endoscopia digestiva, Clínica Farallones. Cali, Colombia.

2 Especialista en patología, Clínica Farallones. Cali, Colombia.

3 Residente de medicina interna, Universidad Libre. Cali, Colombia.

${ }^{4}$ Médico general, Clínica Farallones. Cali, Colombia.

Fecha recibido: $18-05-17$

Fecha aceptado: 22-01-18

\begin{abstract}
Resumen
Se realizó un estudio descriptivo tipo reporte de caso de un paciente valorado por el servicio de gastroenterología en la Clínica Nuestra en la ciudad de Cali, por sospecha de sangrado digestivo oculto cuyos estudios previos no habían mostrado causa de sangrado. Se realizó videocápsula endoscópica (MiroCam ${ }^{\circledR}$ ) con un hallazgo positivo de lesión en el intestino delgado. Se llevó a enteroscopia en Clínica Farallones de la Ciudad de Cali con enteroscopio Olympus 180 Q que confirmó el hallazgo. Ulteriormente, se llevó a cirugía, en la que se confirmó el diagnóstico. Los datos y las fotografías se obtuvieron en bases de datos de los servicios de endoscopia y las fotos de patología se obtuvieron del registro del médico patólogo. Finalmente, se realizó una búsqueda en PubMed sobre literatura existente de historia clínica y reportes de casos similares.
\end{abstract}

\section{Palabras clave}

Hemangioma cavernoso, hemorragia gastrointestinal oculta, cápsula endoscópica.

\section{Abstract}

This is a descriptive study based on the case report of a patient suspected of occult gastrointestinal bleeding who was evaluated by the gastroenterology service in Clínica Nuestra in Cali. Previous studies had not shown cause of bleeding. Video endoscopy (Mirocam) found a lesion in the small intestine. Enteroscopy at performed at Clínica Farallones in Cali with an Olympus $180 \mathrm{Q}$ enteroscope confirmed the finding. Surgery further confirmed the diagnosis. Data and photographs were obtained from the database of endoscopy services and the pathology photos were obtained from the pathologist's registry. A literature review based on a Pubmed search of the existing literature on clinical histories and reports of similar cases is included.

\section{Keywords}

Cavernous hemangioma, occult gastrointestinal hemorrhage, endoscopic capsule.

\section{INTRODUCCIÓN}

Los hemangiomas gastrointestinales son tumores primarios benignos, raros en todos los grupos de edad y representan el $5 \%$ de todos los tumores gastrointestinales (1). Se clasifican en capilares, cavernosos o mixtos; y el cavernoso es el más frecuente (2). Pueden aparecer como un fenómeno aislado o ser parte de trastornos vasculares sistémicos (2).
Entre las posibles formas de presentación se encuentran la anemia, la hemorragia digestiva (el 5\% de los sangrados digestivos se originan en el intestino delgado), el hematoma intramural, la perforación y la obstrucción intestinal; esta última es poco frecuente salvo que sea de gran tamaño y ocluya la luz intestinal (3).

El diagnóstico de esta lesión es difícil; generalmente se necesitan varias pruebas diagnósticas, muchas de ellas 
invasivas (4). Se concluye que solo el $25 \%$ de los casos se diagnostican preoperatoriamente. Tradicionalmente, su diagnóstico se ha basado en la angiografía, el estudio nuclear con gammagrafía con hematíes marcados, la resonancia magnética y la enteroscopia. Esta última, a pesar de ser muy sensible, tiene el inconveniente de que generalmente solo logra explorar unos 80 a $120 \mathrm{~cm}$ distalmente al ángulo de Treitz, además de ser una prueba invasiva, molesta para el paciente y con posibles complicaciones como dolor abdominal postendoscopia, perforación, pancreatitis, entre otras (5).

La cápsula endoscópica es el estándar de oro para identificar las lesiones del intestino delgado (6). Este estudio mejora significativamente el diagnóstico de lesiones vasculares intestinales en comparación con las otras técnicas mencionadas anteriormente (7). Con esta se puede obtener un diagnóstico preoperatorio sin pruebas cruentas y con un bajo riesgo. Otras pruebas invasivas no son eficaces hasta en un 75\% de los casos y, además, la realización repetida de estas conlleva a un mayor costo económico. Hay que destacar que el beneficio de la cápsula endoscópica está en relación con el tamaño de la lesión y la existencia de hemorragia activa en el momento de la exploración.

La resección quirúrgica es el tratamiento de elección. La invaginación intestinal e incluso la perforación causada por el hemangioma cavernoso son extremadamente raros $(8)$ y es muy rara la recurrencia posquirúrgica (9). En aquellos casos de hemangiomas no resecables o difusos, se han utilizado diversos tratamientos con un éxito limitado, como son la radioterapia con dosis bajas, la crioterapia o la embolización arterial (10).

\section{PRESENTACIÓN DEL CASO}

Se trata de un paciente masculino de 67 años quien ingresó al servicio de urgencias por un cuadro de dolor abdominal y rectorragia asociado con síntomas constitucionales: fatiga, astenia y adinamia. Se tomaron paraclínicos en los que se reportó un hemograma con anemia de volúmenes bajos. Al tercer día de hospitalización se evidenció un descenso de hemoglobina de $1 \mathrm{~g} / \mathrm{dL}$ (Tabla 1), por lo que se indicó una transfusión de hemoderivados ( 2 unidades de glóbulos rojos). Se realizó una colonoscopia total en la que no se evidenció ninguna causa de sangrado. Entonces, se indicó la realización de endoscopia de vías digestivas altas que tampoco mostró lesiones que expliquen el sangrado.

Teniendo en cuenta que la endoscopia de vías digestivas altas y en colonoscopia total no mostraron sitio de sangrado, se realizó una cápsula endoscópica, en la que se visualizaron 2 lesiones de aspecto violáceo en el yeyuno proximal, circundante bajo la mucosa intestinal, con aspecto de frambuesa, sin estigmas de sangrado. La impresión diagnóstica

Tabla 1. Laboratorios

\begin{tabular}{lccccc}
\hline \multirow{2}{*}{ Laboratorios } & \multicolumn{5}{c}{ Fechas } \\
\cline { 2 - 5 } & $\mathbf{3 0 - 7 - 1 6}$ & $\mathbf{0 1 - 8 - 1 6}$ & $\mathbf{0 5 - 8 - 1 6}$ & $\mathbf{1 0 - 8 - 1 6}$ & $\mathbf{2 7 - 1 0 - 1 6}$ \\
\hline Leucocitos & 7420 & 6400 & 5360 & 3640 & 3260 \\
Neutrófilos (\%) & 57 & 58,1 & 59,9 & 63,5 & \\
Linfocitos (\%) & 28 & 25,6 & 22,8 & 21,6 & \\
Monocitos (\%) & 8,9 & 10,8 & 10,3 & 8 & \\
Basófilos (\%) & 0,8 & 1,1 & 2,1 & 0,6 & 163 \\
Plaquetas & 444 & 444 & 360 & 221 & $10,5^{* * *}$ \\
Hemoglobina (mg/dL) & 8,1 & 7,6 & $11,7^{*}$ & $10,5^{* *}$ & 1,09 \\
Hematócrito (\%) & 28 & 25,7 & 37 & 78,3 & \\
Volumen corpuscular medio & 69,3 & 69,1 & 75,5 & & \\
Creatinina (mg/dL) & 1,12 & & & & \\
BUN (mg/dL) & 12,4 & & & & \\
PT (s) & 11,5 & & & & \\
PTT (s) & 32,4 & & & & \\
INR & 1,11 & & & & \\
\hline
\end{tabular}

*Hemoglobina postransfusión de hemoderivados.

**Hemoglobina posquirúrgica.

*** Último control de hemoglobina.

BUN: nitrógeno ureico sanguíneo; INR: índice internacional normalizado; PT: tiempo de protrombina; PTT: Tiempo de tromboplastina parcial. 
inicial por cápsula endoscópica fue de tumor neuroendocrino frente a sarcoma de Kaposi (Figura 1).

Ulteriormente, se realizó una enteroscopia anterógrada con balón más marcación con tinta china explorando hasta el yeyuno medio, en la que se observó entre el yeyuno proximal y medio una lesión $\mathrm{de}+/-2 \mathrm{~cm}$ discretamente elevada, hipervascularizada, con compromiso circular, que no dificulta la progresión del equipo. Se realizó la marcación con tinta china con ayuda de aguja endoscópica por encima y por debajo de la lesión (Figuras 2 y 3 ).

El paciente fue llevado a laparoscopia para realizar una resección de yeyuno proximal en la que se encontró un tumor gastrointestinal sin evidenciarse otras lesiones sincrónicas.

La patología reveló en su descripción macroscópica (Figuras 4, 5 y 6) un segmento del intestino delgado que medía $11,5 \mathrm{~cm}$ de largo hasta $3,5 \mathrm{~cm}$ de diámetro, provisto de un mesenterio correspondiente y sin orientación. La mucosa se encontraba evertida y ambos extremos estaban abiertos. La serosa era lisa y se observaron marcaciones con tinta china en el tercio medio. La mucosa mostró una lesión en forma de placa, con pliegues aplanados, de coloración violácea, que medía $2,4 \times 2,2 \mathrm{~cm}$, que mostró al corte microquistes ocupados por sangre. La lesión se localizó a $4 \mathrm{~cm}$ del borde más cercano y a $5,2 \mathrm{~cm}$ del opuesto. El resto de la mucosa intestinal mostró pliegues de aspecto usual y no se encontraron otras lesiones. Las marcaciones con tinta china de la serosa coincidieron con la lesión submucosa. El mesenterio mostró áreas de congestión y no se hallaron ganglios linfáticos. En su descripción microscópica (Figuras 7, 8, 9 y 10), en los cortes histológicos se observó la pared de intestino delgado con una lesión proliferativa, conformada por canales vasculares cavernosos grandes, de paredes delgadas mínimas o fibromusculares, tapizadas por el endotelio sin atipias y ocupadas por sangre. Esta lesión se localizó principalmente al nivel de la submucosa y focalmente se extendió a la lámina propia y hasta la muscular interna. El diagnóstico fue compatible con hemangioma cavernoso.

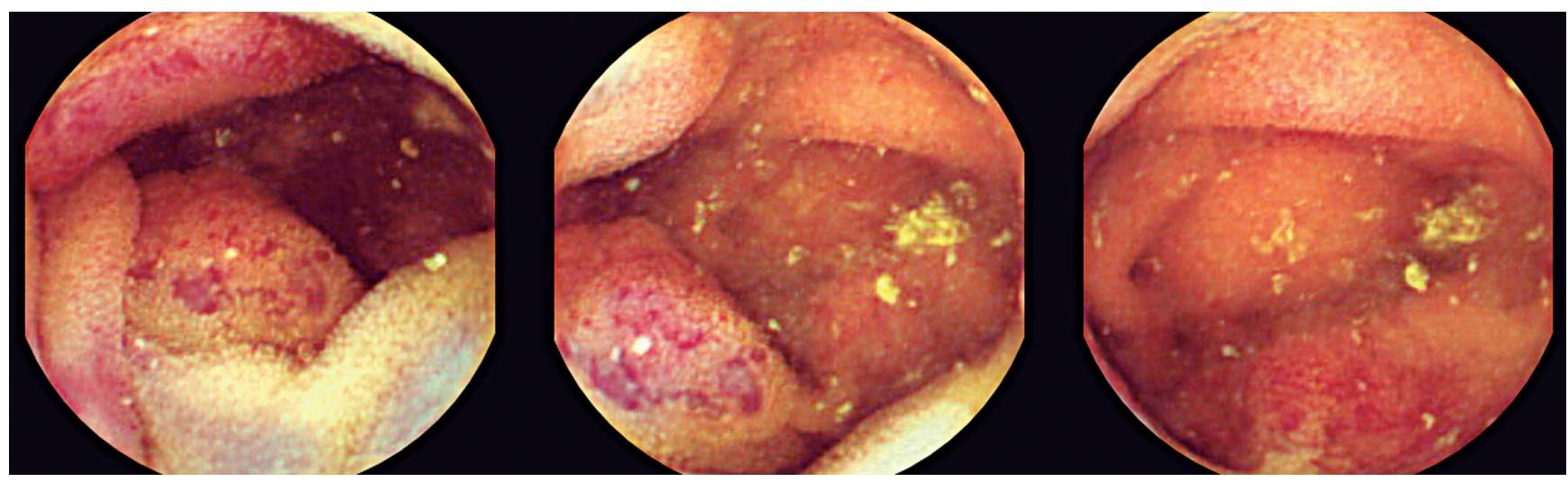

Figura 1. Visualización de hemangioma de intestino delgado por cápsula endoscópica.

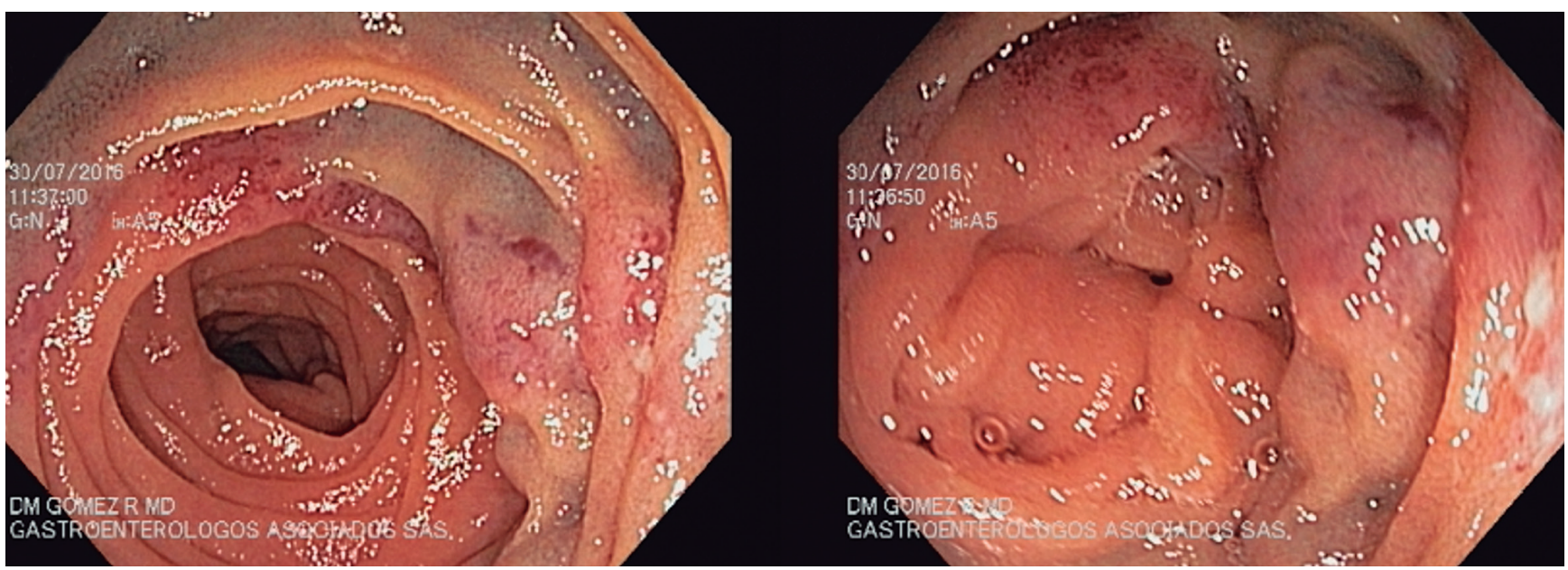

Figura 2. Visualización de hemangioma del intestino delgado por enteroscopia anterógrada con balón. 


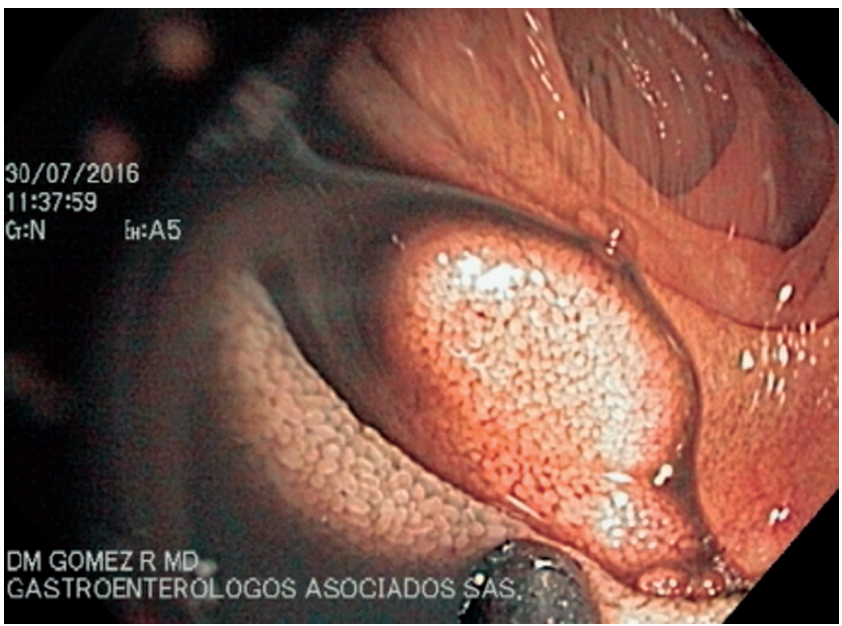

Figura 3. Marcación con tinta china del hemangioma de intestino delgado por enteroscopia anterógrada.

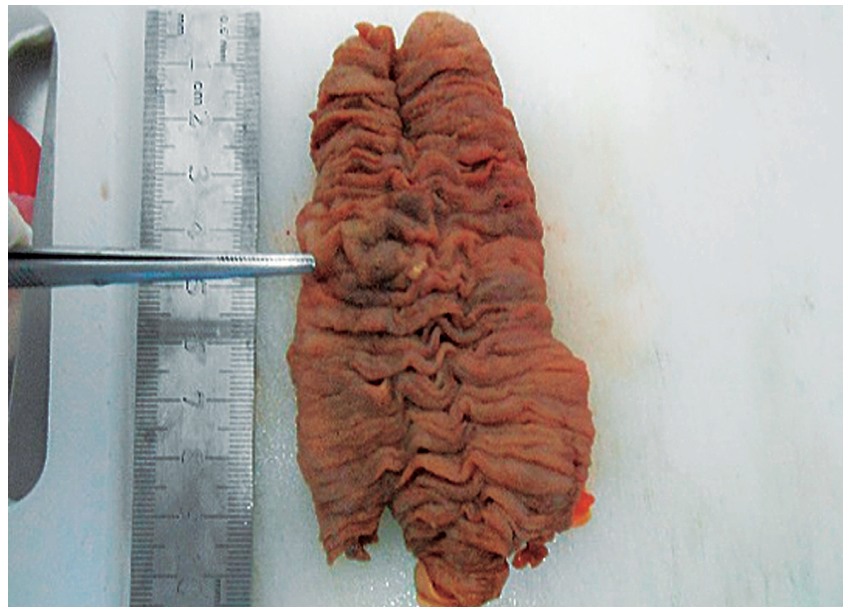

Figura 5. Lesión vascular en mucosa.

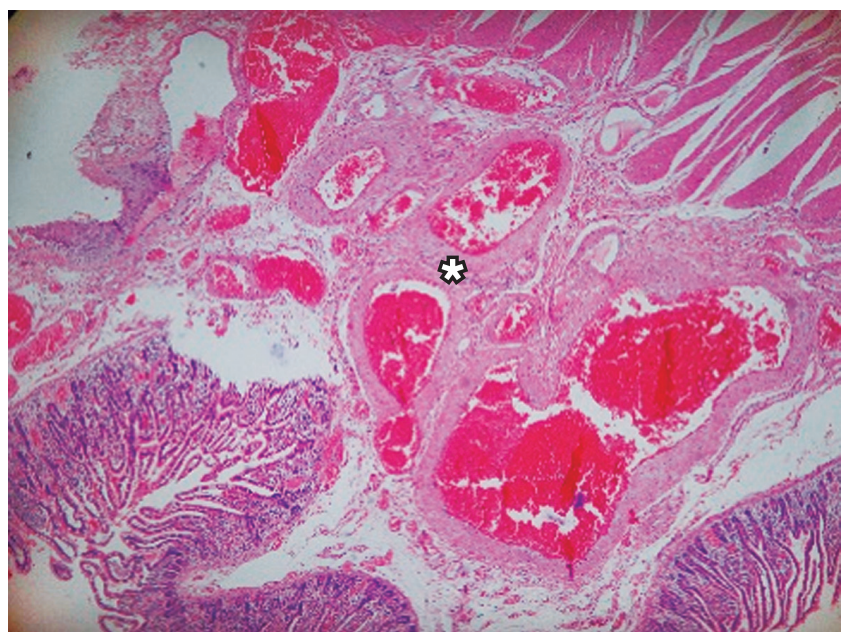

Figura 7. Lesión proliferativa vascular localizada principalmente en la submucosa.

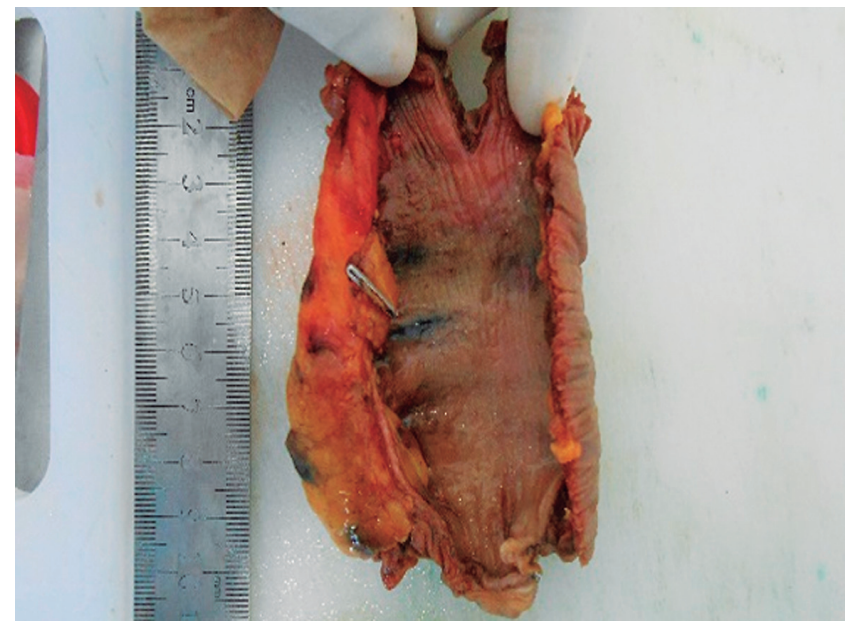

Figura 4. Marcación con tinta china en serosa.

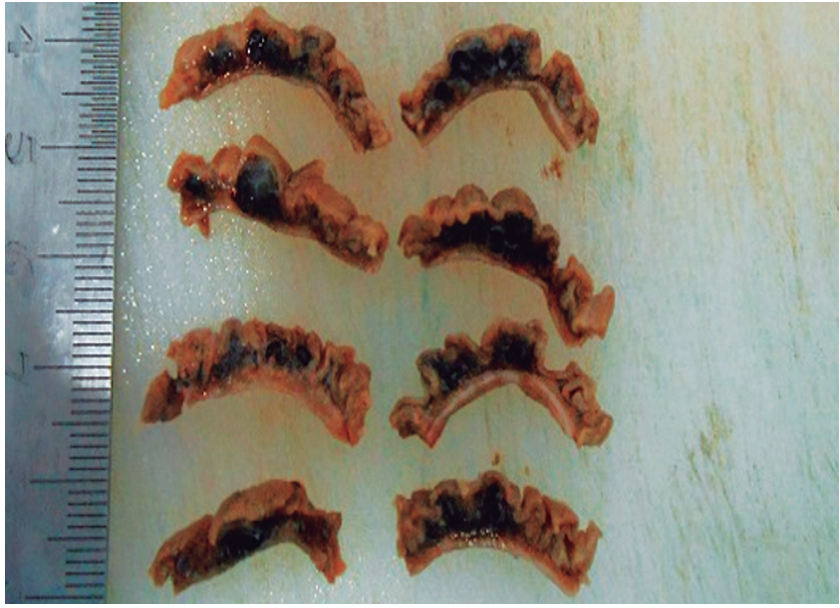

Figura 6. Cortes de la lesión.

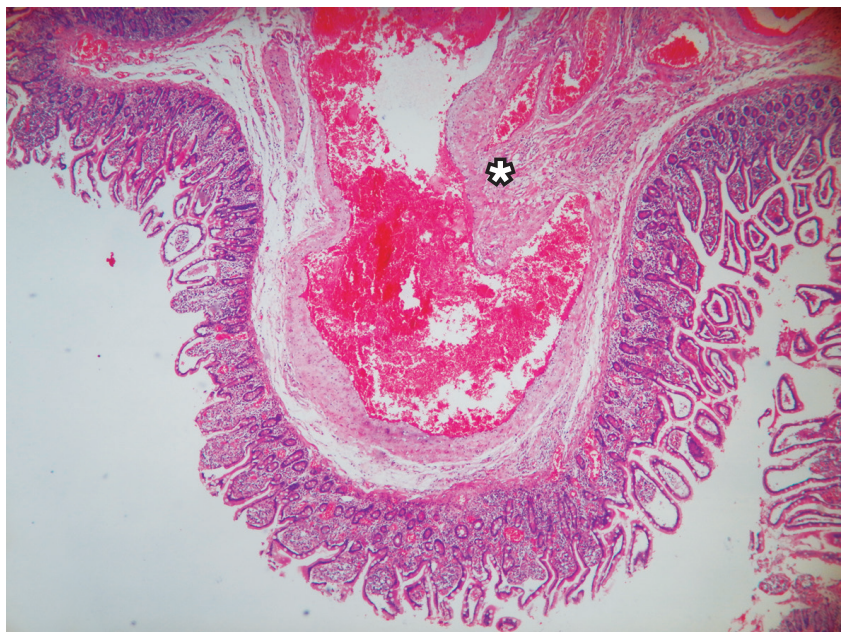

Figura 8. Lesión proliferativa vascular localizada principalmente en la submucosa. 


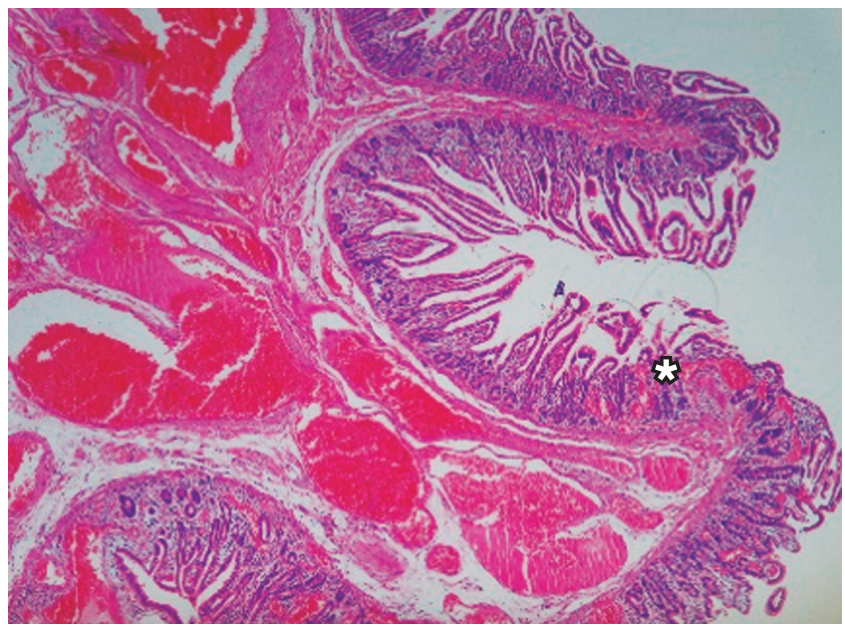

Figura 9. Lesión proliferativa vascular con extensión a lámina propia.

En la actualidad, el paciente se encuentra estable, sin nuevos episodios de sangrado y sin anemia (Tabla 1). Continúa en controles con gastroenterología.

\section{DISCUSIÓN}

Los hemangiomas del tracto gastrointestinal son tumores benignos inusuales que debutan en su mayoría con sangrado. Aunque es una causa poco frecuente de hemorragia gastrointestinal, el hemangioma del intestino delgado a menudo puede producir hemorragia aguda o a anemia crónica y la clínica de melena se observa en el $65 \%$ de los casos. Se presentan con mayor frecuencia en el yeyuno (46\%), aunque pueden comprometer todo el tracto gastrointestinal y manifestarse como masas intramurales o intraluminales. A nivel de colon, son más comunes en el rectosigmoide y pueden tener el aspecto atípico de una lesión anular rígida que imita un carcinoma. La relación de presentación hombre-mujer es igual y la edad media de presentación es de 34,6 años $(1,11)$.

El caso descrito en el presente reporte es comparable con la literatura actual en cuanto a la presentación clínica en pacientes con diagnóstico de hemangioma cavernoso. El cuadro clínico se caracterizó por anemia crónica, pérdida de peso, síntomas constitucionales y sangrado digestivo que llevó al inicio del protocolo por imágenes (12). La endoscopia de vías digestivas altas y la colonoscopia total no revelaron hallazgos compatibles con el origen del sangrado, razón por la cual es importante tener en cuenta esta entidad como una de las etiologías a considerar en los casos de hemorragia digestiva oculta.

La edad media de presentación del caso descrito es inusual: 67 años. La edad media de presentación estable-

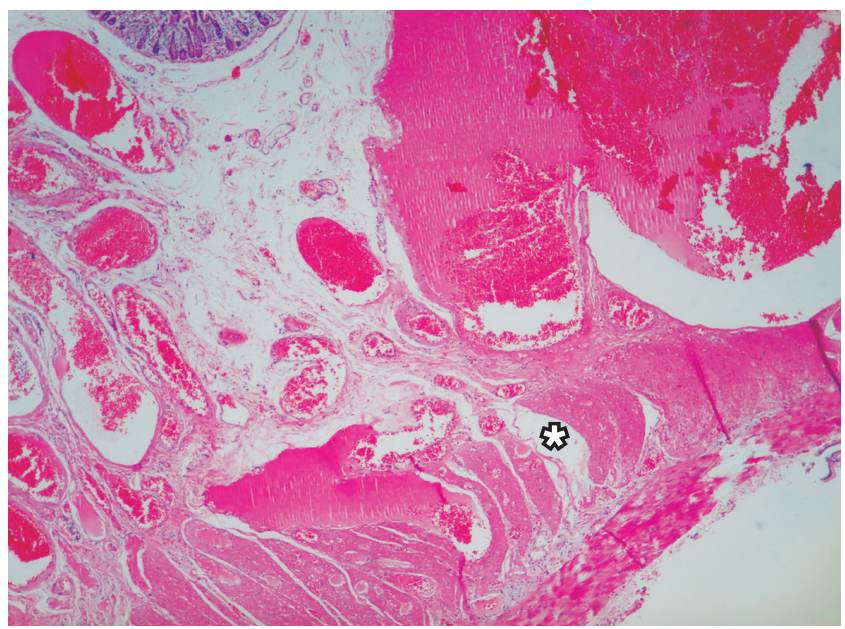

Figura 10. Lesión proliferativa vascular con extensión a muscular interna.

cida según reportes es de 34,6 años, otro punto a tener en cuenta al momento de pensar en alternativas etiológicas en los casos de hemorragia digestiva oculta.

El caso descrito soporta el uso de la cápsula endoscópica como el estándar de oro para sangrado digestivo oculto. El sitio anatómico de presentación documentado por cápsula endoscópica es compatible con la literatura descrita: afección del yeyuno en el $46 \%$ de los casos. La cápsula endoscópica es claramente útil para el diagnóstico preoperatorio de hemangiomas en el intestino delgado.

Para finalizar, este caso muestra cómo la cápsula endoscópica ha revolucionado la evaluación diagnóstica de las lesiones del intestino delgado. Antes de este procedimiento, las lesiones vasculares como el hemangioma cavernoso requerían múltiples investigaciones, a veces invasivas, con productos de diagnóstico incierto y morbimortalidad asociada.

\section{CONCLUSIÓN}

El hemangioma cavernoso es una causa rara de sangrado digestivo oculto proveniente del intestino delgado. En la literatura mundial hay muy pocos casos. El diagnóstico inicial se hace por videocápsula endoscópica o enteroscopia, pero el diagnóstico definitivo y manejo es quirúrgico.

\section{REFERENCIAS}

1. de Mascarenhas-Saraiva MN, da Silva Araújo Lopes LM. Small-bowel tumors diagnosed by wireless capsule endoscopy: report of five cases. Endoscopy. 2003;35(10):865-8. https://doi.org/10.1055/s-2003-42625.

2. Camilleri M, Chadwick VS, Hodgson HJ. Vascular anomalies of the gastrointestinal tract. Hepatogastroenterology. $1984 ; 31(3): 149-53$. 
3. Ell C, Remke S, May A, Helou L, Henrich R, Mayer G. The first prospective controlled trial comparing wireless capsule endoscopy with push enteroscopy in chronic gastrointestinal bleeding. Endoscopy. 2002;34(9):685-9. https://doi. org/10.1055/s-2002-33446.

4. Kuo LW, Chuang HW, Chen YC. Small bowel cavernous hemangioma complicated with intussusception: report of an extremely rare case and review of literature. Indian J Surg. 2015;77(Suppl 1):123-4. https://doi.org/10.1007/ s12262-014-1194-3.

5. Akamatsu T, Matsuda Y, Tsugane E, et al. A case of multiple cavernous hemangioma of the small intestine and clinical review of the Japanese literature. Gastroenterol Jpn. 1990;25(4):494-8.

6. Appleyard M, Fireman Z, Glukhovsky A, et al. A randomized trial comparing wireless capsule endoscopy with push enteroscopy for the detection of small-bowel lesions. Gastroenterology. 2000;119(6):1431-8. https://doi. org/10.1053/gast.2000.20844.

7. Pera M, Márquez L, Dedeu JM, et al. Solitary cavernous hemangioma of the small intestine as the cause of long-standing iron deficiency anemia. J Gastrointest Surg. 2012;16(12):2288-90. https://doi.org/10.1007/s11605-012-1991-6.

8. Pennazio M. Small-intestinal pathology on capsule endoscopy: spectrum of vascular lesions. Endoscopy. 2005 Sep;37(9):864-9. Erratumin: Endoscopy.2005;37(10):1029. https://doi.org/10.1055/s-2005-870212.

9. Donhauser JL, Kelly EC. Intussusception in the adult. Am J Surg. 1950;79(5):673-7. https://doi.org/10.1016/00029610(50)90333-3.

10. Ruiz AR Jr, Ginsberg AL. Giant mesenteric hemangioma with small intestinal involvement: an unusual cause of recurrent gastrointestinal bleed and review of gastrointestinal hemangiomas. Dig Dis Sci. 1999;44(12):2545-51. https:// doi.org/10.1023/A:1026659710815.

11. Allred HW Jr. Hemangiomas of the colon, rectum, and anus. Mayo Clin Proc. 1974;49(10):739-41.

12. Levy AD, Abbott RM, Rohrmann CA Jr, Frazier AA, Kende A. Gastrointestinal hemangiomas: imaging findings with pathologic correlation in pediatric and adult patients. AJR Am J Roentgenol. 2001;177(5):1073-81. https://doi. org/10.2214/ajr.177.5.1771073. 